\title{
Risco de persistência na conduta infracional em adolescentes: estudo exploratório
}

\author{
Persistency risk in the offender conduct in \\ adolescents: exploratory study
}

\author{
Maria Cristina MARUSCHI \\ Ruth ESTEVÃO' \\ Marina Rezende $\mathbf{B A Z O N}^{1}$
}

\begin{abstract}
Resumo
A literatura internacional é consistente em apontar fatores de risco fortemente associados à persistência do comportamento infracional em adolescentes. O conhecimento tem servido de base para o desenvolvimento de instrumentos de avaliação de risco de reincidência infracional, com o propósito principal de adequar a medida judicial, o tipo e o nível de intervenção às necessidades do adolescente. O presente estudo teve como objetivo realizar uma investigação exploratória da performance do instrumento Youth Level of Service/Case Management Inventory, fundamentado nos fatores de risco associados à persistência da conduta infracional, em amostra composta de quarenta adolescentes em conflito com a lei, no interior do Estado de São Paulo. Realizada a coleta de dados inicial, após um período de 6 a 12 meses foi feito levantamento no Cartório da Infância e Juventude para verificar registro de novas infrações. Análises estatísticas mostraram a boa capacidade preditiva do instrumento. Outros resultados e limitações do estudo são apresentados e discutidos.
\end{abstract}

Unitermos: Adolescente. Adolescente em conflito com a lei. Fatores de risco.

\begin{abstract}
The international literature is consistent in pointing risk factors strongly associated with the persistence of offending behavior in adolescents. This knowledge has been used as the basis for the development of instruments to evaluate the risk of recurrent offences, with the main purpose of adapting the judicial measure, type and level of intervention to the needs of the adolescent. The aim of the present study was to carry out an exploratory investigation of the performance of the Youth Level of Service/Case Management Inventory instrument, based on risk factors associated with the persistence of offending behavior, in a samples of forty adolescents in conflict with the law, in the interior of the State ofSão Paulo. After a period of six to twelve months from the initial data collection, a survey was carried out at the Childhood and Youth Registry Office to verify the registration of new offenses. Statistics analysis showed the good predictive capacity of the instrument. Other findings and limitations of the study are presented and discussed.
\end{abstract}

Uniterms: Adolescenct. Adolescence in conflict with the law. Risk factors.

$\boldsymbol{\nabla v \boldsymbol { V } \boldsymbol { v }}$

1 Universidade de São Paulo, Faculdade de Filosofia, Ciências e Letras de Ribeirão Preto, Departamento de Psicologia e Educação. Av. Bandeirantes, 3900 , Monte Alegre, 14040-901, Ribeirão Preto, SP, Brasil. Correspondência para/Correspondence to: M.C. MARUSCHI. E-mail: <cmaruschi@uol.com.br>.

Artigo elaborado a partir da tese da M.C. MARUSCHI, intitulada"Avaliação de adolescente em conflito com a lei a partir dos conceitos de risco e necessidade associados à persistência da conduta infracional". Universidade de São Paulo, 2010. 
As infrações praticadas por adolescentes têm chamado a atenção das pessoas em geral, ocupado grande espaço na mídia e despertado a atenção de pesquisadores de variadas áreas do conhecimento. Em termos práticos, no âmbito do Judiciário, a problemática remete ao desafio cotidiano de lidar diretamente com inúmeros adolescentes em conflito com a lei e de tomar decisões quanto às medidas judiciais cabíveis. As Regras Mínimas das Nações Unidas para a Administração da Justiça de Menores (Gabinete de Documentação e Direito Comparado, 1985), que serviram de base para o Estatuto da Criança e do Adolescente (Brasil, 1990), nesse assunto, preconizam que para a aplicação de medidas socioeducativas o Sistema de Justiça enfatize o bem-estar do adolescente e garanta que qualquer decisão seja não somente proporcional às circunstâncias do infrator e da infração, mas também às circunstâncias e às necessidades tanto do menor de idade quanto da sociedade.

A avaliação das dificuldades e necessidades dos adolescentes, os critérios e as formas utilizados para produzir conhecimento que auxilie a tomada de decisão judicial, no Brasil, variam muito. Isso equivale a dizer que não há uma sistemática que congregue as práticas nesse campo, obtendo-se ao final diferentes entendimentos de Juízes e de Promotores da Infância e Juventude, que podem resultar em diferenças significativas na forma e no rigor da aplicação das medidas. $O$ delito cometido pelo adolescente, sua natureza e gravidade, analisados de acordo com o Código Penal, é, em geral, o dado que mais pesa nas tomadas de decisão judicial.

Em alguns países, como Canadá, Inglaterra, País de Gales, Nova Zelândia, em parte dos Estados Unidos e regiões da Austrália, observa-se um movimento para implantar sistemas em que a coleta e a interpretação de informações sobre o jovem obedeçam a determinados padrões e sejam sistemáticas, permitindo que a medida judicial aplicada seja ajustada às dificuldades e às necessidades do jovem. Isso, para Andrews e Bonta (2006), é elemento fundamental à justiça e ao processo de reabilitação dos adolescentes envolvidos seriamente com a prática de atos infracionais.

Nesse aspecto, a produção de conhecimento científico específico ofereceu uma importante contribuição, entendendo-se como necessário e urgente 680 fomentar também no Brasil a discussão acadêmico- -científica sobre os aspectos que devem ser considerados relevantes na avaliação do adolescente em conflito com a lei e sobre os critérios a serem utilizados para nortear a aplicação de medidas. O presente trabalho tem o propósito de contribuir para tal discussão, relatando uma pesquisa por meio da qual se investigou, de modo exploratório, a pertinência ao contexto brasileiro da aplicação do Youth Level of Service/Case Management Inventory (YLS/CMI) (Inventário de Nível de Serviço para Jovens/Gestão de Caso - Hoge \& Andrews, 2002; 2005). O instrumento, por meio de um check list minucioso, indica níveis de risco de reincidência, com vistas à tomada de decisão sobre as medidas judiciais adaptadas a cada adolescente, na perspectiva de orientar ações de intervenção psicossocial num prisma de prevenção secundária.

O Youth Level of Service/Case Management Inventory é um instrumento canadense, projetado para avaliar fatores de risco e necessidades associados à conduta infracional em adolescentes de 12 a 18 anos. Foi desenvolvido a partir de um instrumento de avaliação para adultos, o Level of Service Inventory-RevisedLSI-R (Andrews \& Bonta, 1995), utilizado em diversas partes do Canadá e também dos Estados Unidos. Teoricamente, o YLS/CMI ancora-se no princípio de que as pessoas diferem no número, tipo, seriedade e variedade de atos antissociais e ilícitos em que se engajam, o que implica em diferenças também nas condições necessárias para reduzir ou abandonar a atividade antissocial/infracional (Andrews \& Bonta, 2006; Bonta \& Andrews, 2007). Nesse sentido, o objetivo principal da avaliação é combinar níveis de intervenção com nível de risco, sendo essa a essência do conceito de risco e a ponte entre a avaliação e o efetivo tratamento.

O Youth Level of Service/Case Management Inventory é, portanto, um instrumento de avaliação de risco e de necessidades, elaborado a partir de estudos de metanálise por meio dos quais se identificaram os fatores com associação mais robusta com a persistência da conduta infracional. Dentre eles, destacam-se quatro fatores com maior força de associação: "atitudes e orientação" (atitudes, valores, crenças e racionalizações que servem de suporte para o comportamento infracional ou "cognição antissocial"), "associação a pares antissociais" (estreita associação com pares envolvidos em atividades ilícitas e relativo isolamento de pares pró- 
-sociais), "história de comportamento antissocial" (envolvimento precoce e contínuo em numerosos e variados atos antissociais, em uma variedade de cenários) e "personalidade/comportamento" (caracterizado pela busca do prazer, fraco controle dos impulsos, baixa autoestima, agressividade e insensibilidade).

Outros quatro fatores, com associação mais fraca, porém significativa, foram também identificados: "situação familiar" (problemas na disciplina e/ou cuidados e na monitoria e/ou supervisão),"educação/emprego" (baixo nível de desempenho e de satisfação na escola e/ou trabalho), "lazer e recreação" (baixo nível de envolvimento e de satisfação em atividades de lazer estruturadas, com orientação de adultos e de natureza pró-social) e o "abuso de álcool e outras drogas".

Os autores chamam atenção para os limites do conhecimento na área, no que se refere, principalmente, aos moderadores específicos que, certamente, fazem variar o impacto dos fatores acima descritos. De qualquer modo, segundo eles, as evidências científicas sobre a importância dos oito fatores indicados é muito sólida, visto que nos estudos de revisão não há investigação - de corte transversal ou longitudinal - em que pelo menos um dos quatro primeiros não seja apontado como fortemente associado à persistência da conduta infracional na adolescência (Andrews \& Bonta, 2006).

A importância dada pela ciência ao estudo dos fatores de risco associados à conduta infracional e o desenvolvimento de instrumentos de avaliação de adolescentes em conflito com a lei dentro dessa perspectiva teórica, em diversas partes do mundo, contrastam com o reduzido número de trabalhos sobre o tema existentes no Brasil, o que justifica o presente estudo. Assim, de modo específico, seu objetivo foi, de um lado, verificar se o YLC/CMI, quando aplicado em adolescentes brasileiros, autores de ato infracional, identificaria adolescentes com diferentes níveis de risco; e, de outro, averiguar a capacidade preditiva do instrumento em relação aos adolescentes avaliados, após o período de 6 a 12 meses da avaliação.

\section{Método}

\section{Participantes}

Participaram da pesquisa 40 adolescentes do gênero masculino, com idade entre 12 e 17 anos (média de 15 anos), e os responsáveis que os acompanhavam, na maioria das vezes a mãe ( $n=25)$, seguida do pai $(n=11)$. Majoritariamente os adolescentes $(n=23)$ estavam evadidos da escola e, entre estes, somente dois tinham concluído o ensino fundamental. Entre os que trabaIhavam ( $n=13$ ), somente cinco continuavam matriculados na escola. No que se refere às infrações praticadas, a maioria remetia a ocorrências de agressão $(n=13)$, seguida de problemas com drogas (porte de entorpecente e tráfico; $n=10)$ e furto qualificado $(n=5)$. Grande parte dos adolescentes estava ingressando no sistema judiciário ( $n=24)$ ou tinha uma única infração anterior $(\mathrm{n}=8)$.

\section{Instrumentos}

Para a coleta de dados foram elaborados roteiros de entrevistas com os adolescentes e com os pais/responsáveis. O roteiro para os pais/responsáveis fundamentou-se no tipo/natureza de informações requeridas para preenchimento do YLS/CMI (Hoge \& Andrews, 2005), enquanto o roteiro para os adolescentes teve como base o guia contido no manual do usuário do referido instrumento. Outra fonte de informação utilizada para o preenchimento do YLS/CMI foi a análise documental dos processos, especialmente no que se refere a atos infracionais anteriores e medidas socioeducativas aplicadas. As informações coletadas nas entrevistas e nos documentos analisados serviram de complementação e/ou confirmação entre si.

Na sequência, conforme mencionado, empregou-se o YLS/CMI, que é, de fato, um check list. A primeira seção do instrumento é composta de 42 itens organizados em oito subescalas, contendo: cinco itens na subescala infrações anteriores, seis itens na subescala situação familiar/parental, sete itens na subescala educação/emprego, quatro itens na subescala relação com os pares, cinco itens na subescala uso de drogas, três itens na subescala lazer/recreação, sete itens na subescala personalidade/comportamento e cinco itens na subescala atitudes/orientação.

Há também um local para indicar "pontos fortes" (fatores protetivos) relevantes para cada adolescente e uma parte para registro dos chamados fatores de responsividade, fundamentais para o desenvolvimento do plano de intervenção. A presente pesquisa, no entanto, ateve-se especificamente à análise dos fatores de risco. 
A aplicação do Youth Level of Service/Case Management Inventory implica marcar cada item da subescala que se aplique ao adolescente, ou seja, se a característica ou circunstância referida está presente para o adolescente avaliado, sendo esses indicadores do fator de risco representados pela subescala. Os itens marcados são somados em cada subescala, obtendo-se o subescore e identificando-se, na sequência, em tabela específica no manual do instrumento, o nível de risco para cada subescala, se "baixo", "moderado" ou "alto".

A soma dos oito subescores fornece o nível de risco global que, a partir de tabela específica do manual, classifica o adolescente em quatro níveis de risco: "baixo", "moderado", "alto" ou "muito alto". Concebe-se que o nível global de risco apresentado pelo jovem é importante para refletir sobre aspectos de segurança e sobre o "nível de serviço" a ser-lhe fornecido, enquanto os subescores são mais relevantes para o planejamento da intervenção.

No que diz respeito às qualidades psicométricas do YLS/CMI, no manual citam-se vários estudos de validade concorrente e preditiva, confiabilidade, concordância entre juízes, correlação do escore global de nível de risco com vários índices de distúrbios comportamentais, sendo os resultados sempre bastante positivos, principalmente para o gênero masculino.

Concernindo estudos realizados em contextos socioculturais diferentes do de origem, destacam-se aqui o de Chesta (2008), no Chile, que analisou a consistência interna, confiabilidade interavaliadores, validade concorrente e discriminante; o de Marshall, Egan, English e Jones (2006), no Reino Unido, que testaram a validade concorrente; e o de Graña, Garrido e Gonzáles (2007), na Espanha, que verificaram a validade preditiva. Todos obtiveram resultados convergentes com estudos anteriores.

\section{Procedimentos}

A pesquisa foi primeiramente analisada e aprovada pelo Comitê de Ética em Pesquisa com Seres Humanos da Faculdade de Filosofia, Ciências e Letras de Ribeirão Preto (FFCLRP) da Universidade de São Paulo (USP) (Protocolo n 397/2008 - 2008.1.1269.59.2). Assim, a coleta de dados, realizada por um único pesquisador, aconteceu mediante assinatura, pelos pais/responsáveis, do Termo de Consentimento Livre e Esclarecido.

A coleta de dados consistiu na realização de entrevistas individuais com os pais/responsáveis, seguidas de entrevistas com os adolescentes, todas gravadas, além de análise documental, por meio de busca e verificação nos processos de informações relevantes.

A coleta de dados ocorreu no período de julho de 2008 a setembro de 2009, por ocasião da apresentação dos adolescentes para oitiva informal junto ao Ministério Público, no fórum de uma comarca do interior do estado de São Paulo. No período compreendido entre maio de 2009 e março de 2010, foi realizada, junto ao Cartório da Infância e Juventude, uma busca por informação relativa à existência ou não de ocorrência de novos envolvimentos infracionais dos adolescentes da amostra, respeitando-se para cada caso o período mínimo de seis meses e máximo de doze, transcorridos da data da coleta de dados.

As informações sobre cada adolescente, colhidas no primeiro momento da coleta de dados por meio dos diferentes procedimentos, foram integradas de modo a compor uma síntese. Com base na síntese, procedeu-se ao preenchimento do YLS/CMI. A partir dos escores obtidos para cada adolescente nas subescalas e no escore global, bem como da identificação do nível de risco conforme instruções do manual do YLS/CMI, foram realizadas análises exploratórias para caracterização da amostra.

Utilizou-se o cálculo do coeficiente de correlação não paramétrica de Spearman para avaliar o peso de cada subescala para o escore global de nível de risco. Com base nas informações relativas ao nível de risco de cada adolescente e no fato de ter ou não reincidido entre seis meses e um ano depois, procedeu-se ao cálculo da curva Receiver Operating Characteristic (ROC), utilizado para verificar a capacidade do instrumento em identificar corretamente os adolescentes que efetivamente apresentaram a reincidência (sensibilidade), bem como aqueles que não apresentaram (especificidade). Com o objetivo de averiguar se a variável escore global de nível de risco do YLS/CMI diferenciava o grupo formado por adolescentes que haviam reincidido dos que não haviam, utilizou-se o teste não paramétrico de 
Mann-Whitney, empregado também para verificar a mesma capacidade em cada uma das subescalas.

\section{Resultados}

A verificação do percentual de adolescentes da amostra segundo o escore global de nível de risco mostrou que $75 \%$ deles encontrar-se-iam nos níveis de risco "muito alto", "alto" e "moderado", ou seja, segundo a avaliação, trinta adolescentes apresentariam maior probabilidade de continuar infringindo as leis se as condições avaliadas se mantivessem inalteradas.

O coeficiente de correlação entre os subescores obtidos em cada uma das oito subescalas do YLS/CMI e o escore global de nível de risco foi diretamente proporcional e estatisticamente significante $(p<0,001)$, demonstrando que todas as subescalas do instrumento mediram o constructo a que se propõem: "infrações anteriores" $(r=0,50)$, "situação familiar/parental" $(r=0,75)$, "educação/emprego" ( $r=0,68)$, "relação com pares" $(r=0,74)$, "uso de drogas" ( $r=0,64)$, "tempo livre" ( $r=049)$, "personalidade/comportamento" $(r=0,58)$ e "atitudes/orientação" $(r=0,78)$.

Utilizou-se o cálculo da curva ROC para aferir a capacidade do YLS/CMI em identificar corretamente os adolescentes que apresentavam risco significativo de persistência infracional em relação aos que efetivamente apresentaram esta conduta entre seis meses e um ano depois da avaliação (sensibilidade), bem como para identificar aqueles que foram classificados no risco baixo e que não reincidiram (especificidade). Optando-se por adotar o ponto de corte 18, o instrumento atingiu nível de sensibilidade de 76,9\% (identificação correta dos adolescentes que apresentaram o problema) e de $66,7 \%$ de especificidade (exclusão correta dos adolescentes que não apresentaram o problema). Isso resultou em uma área sob a curva estatisticamente significante (área=80,8\%, $p=0,002$ ). É importante destacar que na amostra, considerando os níveis de risco propostos, o YLS/CMI discriminou acertadamente 100,0\% dos adolescentes avaliados como apresentando "baixo risco", visto que nenhum deles voltou a cometer infração, de acordo com as informações disponibilizadas no Cartório da Infância e Juventude, conforme pode ser verificado na Figura1.

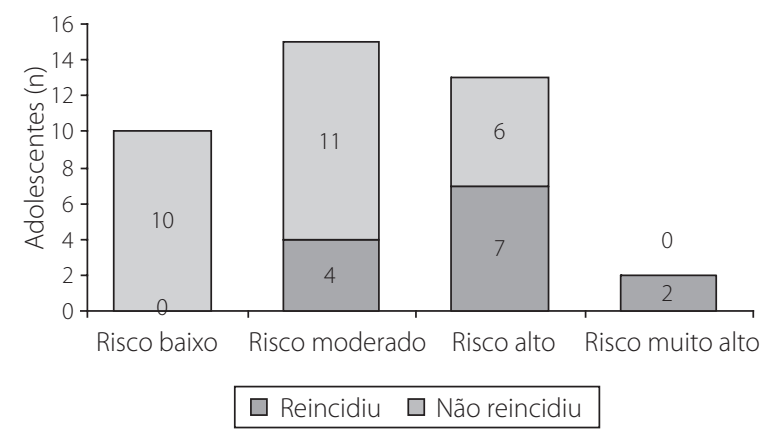

Figura 1. Distribuição do escore global de nível de risco do YLS/CMI e índice de reincidência no comportamento infracional.

Nota: Youth Level of Service/Case Management Inventory.

Com o objetivo de avaliar a capacidade preditiva do YLS/CMI, compararam-se os subgrupos de adolescentes, formados com base no dado referente à reincidência infracional, com o escore global de nível de risco do instrumento. O resultado $(Z=-3,31 ; p=0,001)$ indicou que o instrumento discriminou os dois grupos de adolescentes em relação à probabilidade de reincidência infracional.

Para identificar as subescalas que isoladamente discriminavam os dois grupos de adolescentes, os que reincidiram dos que não reincidiram, verificou-se que aquelas que melhor os discriminaram foram "atitudes/orientação" $(Z=-3,02 ; p<0,01)$, "relação com pares" $(Z=-2,77 ; p<0,01)$, "uso de drogas" $(Z=-2,74 ; p<0,01)$ e"infrações anteriores e atuais" $(Z=-2,31 ; p<0,05)$. As demais subescalas, "tempo livre/recreação" $(Z=-1,73)$, "situação familiar/parental" (Z=-1,57), "personalidade/comportamento" $(Z=-1,42)$ e "educação e emprego" $(Z=-0,95)$ não apresentaram resultados estatisticamente significantes.

\section{Discussão}

Deve-se ressaltar inicialmente que, na amostra pesquisada, o YLS/CMI foi capaz de identificar adolescentes apresentando diferentes níveis de risco para a persistência da conduta infracional. Nesse tocante, uma significativa proporção - 75\% dos adolescentes avaliados - apresentaria risco moderado, alto e muito alto para a persistência infracional. Numa primeira instância, tal resultado parece contrastar com aquilo que era esperado, considerando o contexto de recrutamento, onde predominam adolescentes sem histórico oficial anterior 
de cometimento de ato infracional ( $n=24)$, ou com apenas uma infração anterior $(n=8)$, de menor gravidade.

Do ponto de vista da avaliação realizada com o YLS/CMI, no entanto, com base nos conceitos de risco e de necessidades associadas à "delinquência juvenil", esses adolescentes, apesar daquelas características, apresentariam determinados problemas/dificuldades em aspectos desenvolvimentais e/ou situacionais/contextuais, significativos em relação à problemática, embora a maior parte estivesse iniciando o envolvimento em infrações (75\%). Os problemas/dificuldades, na perspectiva adotada, se não trabalhados por meio de intervenções de ajuda/acompanhamento, aumentam a probabilidade de novos envolvimentos infracionais.

Esse quadro deixa claro que o delito em si é apenas uma dimensão da análise do problema e, portanto, as avaliações feitas no âmbito da justiça, para as tomadas de decisão quanto às medidas socioeducativas, precisam ir além desse aspecto. É importante lembrar que pesquisas indicam que um percentual elevado de adolescentes pratica infrações (em sua maioria pouco sérias), mas que apenas alguns poucos adolescentes se engajam em uma trajetória persistente, tendo iniciado o percurso também com infrações de natureza leve, as quais, com o tempo, vão se intensificando em número e em gravidade (Andrews \& Bonta, 2006; Fréchette \& Le Blanc, 1987, Moffitt, 1993).

O número de infrações anteriores oficialmente conhecidas, indicador para a apreciação da reincidência no âmbito das agências de controle, no contexto da avaliação de risco é considerado um item relevante por indicar processos, como o de rotulação, por exemplo, que por si incrementam a probabilidade de persistência da conduta delituosa (Andrews \& Bonta, 2006; Andrews, Bonta \& Wormith, 2006). Isso evidencia, novamente, a complexidade dos processos que articulam os indicadores de risco (histórico de infrações anteriores, por exemplo) e o efeito associado (no caso do presente estudo, a persistência da conduta).

No que se refere à capacidade preditiva do YLS/CMI, conforme se nota na Figura 1, chama atenção primeiramente o fato de $100 \%$ dos casos avaliados nos níveis de risco considerados extremos (baixo e muito alto) terem confirmado a predição no período de seis meses a um ano depois da avaliação. O fato de 25\% da

684 amostra (dez adolescentes) ter sido classificada no nível de baixo risco e nenhum deles ter efetivamente se envolvido em nova infração, implica pensar na existência de uma proporção de jovens frente aos quais se deveriam adotar, idealmente, procedimentos jurídicos e institucionais breves e leves, de modo a, inclusive, evitar os efeitos da rotulação e da socialização nas instituições de controle.

Para Andrews e Bonta (2006), diferentemente dos infratores classificados no nível de risco alto, que tendem a responder de maneira mais favorável a um formato de tratamento intensivo, para os adolescentes de baixo risco um medida judicial que crie um quadro de intervenção maciça e intensa pode não ter efeito algum e, inclusive, pode produzir efeitos negativos no desenvolvimento. Posto isso, verifica-se que a ideia principal do YLS/CMI é a de combinar a aferição do risco com o nível de intervenção, além de identificar as áreas que precisam de intervenção, com vistas à redução do risco (Hoge \&Andrews, 2005).

É certo que a proposta de avaliação de níveis de risco pode suscitar o problema ético relacionado ao perigo de predizer problemas no comportamento humano, e assim, fomentar profecias autorrealizadoras, ainda que se faça a ressalva de que predições no plano científico nunca são perfeitas. De todo modo, na prática cotidiana do Sistema de Justiça Juvenil, a partir do momento em que o jovem é considerado autor de um ato infracional, ele é avaliado e, a partir daí, tomam-se decisões que afetam de forma concreta e radical sua vida, porque em algum nível se formaram convicções sobre suas dificuldades e necessidades.

Assim, parece melhor que as avaliações incluam métodos e técnicas baseadas em fundamentação científica sólida. Nesse sentido, os resultados de três décadas de pesquisas forneceram evidências sobre os fatores associados ao aparecimento e ao incremento da problemática do engajamento de adolescentes em atividades ilegais/criminais, sendo que isso pode orientar a aplicação de medidas e a execução de programas eficazes (Lavoie, Guy \& Douglas, 2009).

Na amostra pesquisada, o cálculo da Curva ROC demonstrou que o instrumento atingiu níveis aceitáveis de sensibilidade e de especificidade. Pode-se dizer, considerando o propósito do instrumento, que os índices de discriminação (sensibilidade e especificidade) encontrados são satisfatórios para os objetivos a que se 
propõem, uma vez que conseguem identificar com razoável segurança o nível de risco e as necessidades mais prevalentes nos adolescentes.

Confirmando também as qualidades psicométricas do instrumento, os resultados obtidos indicam que ele foi capaz de discriminar os adolescentes que reincidiriam daqueles que não reincidiriam, sendo as subescalas "atitudes/orientação", "relação com pares", "uso de álcool e outras drogas" e "histórico de infrações anteriores e atuais" as mais importantes nesse sentido. Os resultados convergem com estudos internacionais realizados com o mesmo propósito. Entre essas quatro subescalas que mais teriam contribuído para a identificação do nível de risco junto à amostra estudada, três seriam fortemente associadas à persistência da conduta delituosa, de acordo com a metanálise realizada por Andrews e Bonta (2006): "atitudes/orientação", "relação com pares" e "histórico de infrações anteriores e atuais", corroborando os estudos que sempre apontam ao menos uma delas como sendo a dimensão de maior peso. Destaca-se que, dentre cinco estudos que avaliaram a capacidade discriminante do YLS/CMI (Hoge \& Andrews, 2005; Chesta, 2008; Graña et al., 2007), quatro apontam as subescalas "atitudes/orientação"e "relação com pares" como os melhores preditores.

Assim, salta aos olhos a convergência dos estudos em torno das subescalas "atitudes/orientação" e "relação com pares" como melhores preditores para o repetição do comportamento infracional. É interessante, portanto, pensar no significado dos problemas aos quais remetem essas duas subescalas, em separado e conjuntamente. Estudos mostram que atitudes e crenças divergentes, durante a adolescência, aumentam a probabilidade de associação com pares também divergentes, aumentando o risco de atividade divergente - incluindo o cometimento de delitos -, o que, por sua vez, consolida as atitudes e as crenças divergentes (Butler, Leschied \& Fearon, 2007). Ademais, sabe-se que o relacionamento com pares na adolescência cria um contexto de socialização no qual comportamentos, atitudes, crenças e valores também são aprendidos/adotados, o que, por seu turno, reforça a afiliação, devido à identificação (Haynie, 2002). Assim, estar-se-ia diante de um nítido processo de retroalimentação entre esses dois fatores.
Considerando que, no contexto em que a presente pesquisa foi realizada, essas seriam as duas subescalas mais importantes na discriminação dos adolescentes que reincidiram e que não reincidiram, o processo deveria ocupar um lugar central nas preocupações dos agentes socioeducativos, no sentido de pensar quais medidas deveriam ser implementadas para interferir nessa dinâmica. Vale explicitar que, no âmbito das "atitudes e orientação", aquilo que parece contar prioritariamente para que a conduta delituosa se repita é a denominada "cognição antissocial". Esta implica em desvalorizar o respeito às leis e às regras sociais, junto a uma valorização da utilidade do ilícito/crime, com base em racionalizações que justificam a violação à lei e a identificação com valores criminais, acrescidas da presença de sentimentos de raiva, ressentimento e adoção de uma postura desafiadora (Andrews \& Bonta, 2006; Hoge \& Andrews, 2005). No que se refere ao "relacionamento com pares", deve-se lembrar que o problema remete não somente à associação a pares divergentes, mas ao afastamento de pares pró-sociais, o que, segundo a literatura, aumenta a probabilidade de persistência do comportamento no final da adolescência, período em que a conduta infracional tenderia à redução, por si mesmo, se estes fatores não estivessem presentes (Andrews \& Bonta, 2006; Farrington, Jolliffe, Loeber, Stouthamer-Loeber \& Kalb, 2001; Shortt, Capaldi, Dishion, Bank \& Owen, 2003; Woodward, Fergusson \& Horwood 2002).

O terceiro fator que melhor discriminou o grupo de adolescentes reincidentes dos não reincidentes, o "uso de álcool e outras drogas", congrega itens referentes ao grau com que se usam essas substâncias, com vistas à caracterização do consumo, no interesse de identificar o uso abusivo. Na amostra estudada, os adolescentes que reincidiram, em boa parte, apresentariam essa problemática, confirmando apontamentos da literatura sobre a relação entre uso abusivo de álcool/outras drogas e conduta infracional na adolescência e, mais especificamente, com o fenômeno da persistência (Heim \& Andrade, 2008; Slade et al., 2008; Stadtland \& Nedopil, 2003; Stoolmiller \& Blechman, 2005). Vale frisar, segundo Mason, Hitchings, McMahon e Spoth (2007), que, uma vez instalada a conduta infracional, ela própria desencadeia uma sequência de situações, contextos e eventos que podem comprometer o funcionamento adaptativo, 
aumentando a probabilidade de uso de álcool e outras drogas, o que, por seu turno, incrementa a probabilidade de persistir o comportamento infracional.

A quarta subescala,"infrações anteriores e atuais", busca verificar a existência ou não de um histórico de infrações reconhecidas oficialmente e se houve ou não aplicações de medidas socioeducativas anteriores. Embora tal aspecto não remetesse a priori a uma característica marcante na amostra, conforme anteriormente sublinhado, a análise mais específica indicou ser esse um aspecto relevante na discriminação dos grupos de adolescentes - aqueles que reincidiram e aqueles que não reincidiram -, o que corrobora apontamentos da literatura sobre o peso dessa variável na persistência da conduta delituosa na adolescência (Andrews \& Bonta, 2006; Fréchette \& Le Blanc, 1987; Moffitt, 1993).

Com base no conjunto dos resultados obtidos, esboça-se, para o grupo dos adolescentes estudados e especificamente para os reincidentes, a existência de uma dinâmica geradora de problemas, favorecedora da persistência da conduta infracional. Essa conduta se assentaria no fato de eles apresentarem atitudes e orientação antissociais, provavelmente adquiridas e/ou reforçadas no relacionamento com os pares, com os quais também estariam aprendendo e/ou sentindo-se reforçados no uso abusivo de álcool e outras drogas, o que, por seu turno, engendraria motivações e/ou ocasiões para o cometimento de novos atos infracionais. $\mathrm{O}$ fato de ser flagrado pela polícia e submetido aos procedimentos da justiça mais de uma vez (construindo assim um histórico de infrações oficialmente conhecidas e sancionadas) acrescentar-se-ia à dinâmica, reforçando para os próprios adolescentes uma imagem negativa de si mesmos, a qual, ao longo do tempo, pode ser introjetada a ponto de repercutir no desenvolvimento da personalidade e, por conseguinte, na formação identitária.

\section{Considerações Finais}

Considerando ser o presente trabalho o primeiro de que se têm notícia no Brasil, empregando o instrumento de avaliação YLS/CMI, seus resultados devem ser tomados com parcimônia, relembrando o caráter exploratório do mesmo. De todo modo, o estudo traz informações interessantes sobre o instrumento e, conse- quentemente, sobre a pertinência dos conceitos a ele subjacentes, considerando o fenômeno em foco e a realidade brasileira.

Obviamente, vários dos apontamentos feitos remetem à necessidade de novos estudos, inclusive no sentido de superar algumas das limitações desta pesquisa, como, por exemplo, o tamanho reduzido da amostra. Outro aspecto a ser considerado refere-se ao local onde se recrutou a amostra estudada (o fórum), visto que dele geralmente se encontram excluídos os adolescentes com uma trajetória infracional oficial comprovadamente mais séria ou recorrente. Em geral, quando identificados em infrações novas, esses adolescentes são detidos em unidades de internação provisória, até que o juiz decida pela aplicação de alguma medida socioeducativa. Outros estudos, com diferentes amostragens - inclusive com adolescentes multirreincidentes, com perfil infracional bem diferente da amostra aqui estudada - podem ampliar o conhecimento sobre as qualidades psicométricas do instrumento e possibilitar análises e informações complementares.

\section{Referências}

Andrews, D. A., \& Bonta, J. (1995). The level of service inventoryrevised. Canadá: Multi-Health Systems.

Andrews, D. A., \& Bonta, J. (2006). The Psychology of criminal conduct ( $4^{\text {th }}$ ed.). Canadá: LexixNexis. (Original work published 1994).

Andrews, D. A., Bonta, J., \& Wormith, J. S. (2006). The recent past and near future of risk and/or need assessment. Crime \& Delinquency, 52 (1), 7-27.

Bonta, J., \& Andrews, D. A. (2007). Risk-need-responsivity model for offender assessment and rehabilitation. Ottawa: Public Safety Canada. Retrieved on January 27, 2012, from <www.publicsafety.gc.ca/res/cor/rep/risk_need_ 200706-eng.aspx>.

Brasil. (1990). Lei no 8069, de 13 de julho de 1990. Dispõe sobre o Estatuto da Criança e do Adolescente e dá outras providências. Brasília: Presidência da República.

Butler, S. M., Leschied, A. W., \& Fearon, P. (2007). Antisocial beliefs and attitudes in pre-adolescent and adolescent youth: the development of the antisocial beliefs and attitudes scales (ABAS). Journal of Youth and Adolescence, 36 (8), 1058-1071.

Chesta, S. A. (2008). Características psicométricas del inventario de riesgos y necessidades vinculados con factores criminogénicos (IRBC). Dissertação de mestrado não-publicada, Universidade de la Frontera, Temuco, Chile. 
Farrington, D. P., Jolliffe, D., Loeber, R., Stouthamer-Loeber, M., \& Kalb, L. M. (2001). The concentration of offenders in families, and family criminality in the prediction of boys' delinquency. Journal of Adolescence, 24 (5), 579-596.

Fréchette, M., \& Le Blanc, M. (Orgs). (1987). Délinquances et Délinquants. Montreal: Gaëtan Morin Éditeur.

Gabinete de Documentação e Direito Comparado. (1985). Regras mínimas das Nações Unidas para administração da justiça da infância e da juventude (Regras de Beijing). Resolução 40/33, de 29 de novembro de 1985. Recuperado em maio 24, 2009, disponível em <http://www. gddc.pt/direitos-humanos/textos-internacionais-dh/ tidhuniversais/dhaj-NOVO-regrasBeijing.html>.

Graña, J. L., Garrido, V., \& Gonzáles, L. (2007). Evaluación de las características delictivas de menores infractores de la comunidad de Madrid y su influencia em la planificación del tratamiento. Psicopatologia Clínica Legaly Forense, 7, 7-18.

Haynie, D. L. (2002). Friendship networks and delinquency: the relative nature of peer delinquency. Journal of Quantitative Criminology, 18 (2), 99-134.

Heim, J., \& Andrade, A. G. (2008). Efeitos do uso do álcool e das drogas ilícitas no comportamento de adolescentes de risco: uma revisão das publicações científicas entre 1997 e 2007. Revista de Psiquiatria Clínica, 35 (Supl. 1), 61-64.

Hoge, R. D., \& Andrews, D. A. (2002). Youth level of servicel case management inventory: user's manual. Toronto: Mult-Health Systems Inc.

Hoge, R. D., \& Andrews, D. A. (2005). Youth level of service: case management inventory: user's manual. Canadá: MultHealth Systems Inc.

Lavoie, J. A. A., Guy, L. S., \& Douglas, K. S. (2009). Violence risk assessment: principles and models bridging prediction to management. In J. L. Ireland, C. A., Ireland \& P. Birch (Eds.), Violent and sexual offenders. New York: Willan Publishing.
Marshall, J., Egan, V., English, M., \& Jones, R. M. (2006). The relative validity of psychopathy versus risk/needs-based assessments in the prediction of adolescent offending behavior. Legal and Criminological Psychology, 11 (2), 197-210.

Mason, W. A., Hitchings, J. E., McMahon, R. J., \& Spoth, R. L. (2007). A test of three alternative hypotheses regarding the effects of early delinquency on adolescent psychosocial functioning and substance involvement. Journal Abnormal Child Psychology, 35 (5): 831-843.

Moffitt, T. E. (1993). Adolescence-limited and life-coursepersistent antisocial behavior: a developmental taxonomy. Psychological Review, 100 (4), 674-701.

Shortt, J. W., Capaldi, D. M., Dishion, T. J., Bank, L., \& Owen, L. D. (2003). The role of adolescent friends, romantic partners, and siblings in the emergence of the adult antisocial lifestyle. Journal of Family Psychology, 17 (4), 521-533.

Slade, E. P., Stuart, E. A., Salkever, D. S., Karakus, M., Green, K. M., \& lalongo, N. (2008). Impacts of age of onset of substance use disorders on risk of adult incarceration among disadvantaged urban youth: a propensity score matching approach. Drug Alcohol Dependence, 95 (1-2), 1-13.

Stadtland, C., \& Nedopil, N. (2003). Alcoholl and drogs and their relation to criminal recidivism. Fortschritte der Neurologie Psychiatrie, 71 (12), 654-660.

Stoolmiller, M., \& Blechman, E. A. (2005). Substance use is a robust predictor of adolescent recidivism. Criminal Justice and Behavior, 32 (3), 302-328.

Woodward, L. J., Fergusson, D. M., \& Horwood, L. J. (2002). Deviant partner involvement and offending risk in early adulthood. Journal of Child Psychology and Psychiatry, 43 (2), 177-190

Recebido em: 14/4/2011

Aprovado em: 16/2/2012 
